\title{
STUDIES OF LUMINESCENCE.
}

By Edward L. Nichols and Ernest Merritt.

\section{The Luminescence of Sidot Blende. ${ }^{1}$}

$A$ MONG the substances whose luminescence is sufficiently bright A for spectrophotometric study Sidot blende, or phosphorescent zinc sulphide, seems especially well suited to bring out the relationships that doubtless exist between different types of luminescence ; for not only is this substance excited to luminescence by all known exciting agents - light, Roentgen rays, radium rays, cathode rays, etc. - but the stimulating effect of heat, and the property possessed by the red and infra-red rays of suppressing phosphorescence, are exhibited in unusual degree. It is for this reason that we have chosen this substance for spectrophotometric study. While it is our intention to extend this study to all of the various types of luminescence whose quantitative investigation is practicable, the present communication deals only with luminescence excited by light and by Roentgen rays.

The material used in these experiments was in the form of a screen, of the kind frequently used in exhibiting the properties of radium and for numerous experiments in which it is desirable to have a fluorescent substance in convenient form. The experimental methods were similar to those employed in our previous work on fluorescence, a Lummer-Brodhun spectrophotometer, with an acetylene flame as a comparison source, being used to measure the intensity in different parts of the luminescence spectrum. In certain portions of the work special devices were required which will be described in their proper place.

\section{Luminescence Excited by Roentgen Rays.}

The screen was placed in front of the collimator slit of the spectrophotometer at a distance of only a few centimeters, while the

t An account of the experiments described in this paper was presented to the American Physical Society at the Philadelphia meeting December 30, 1904. An abstract appeared in the Physical Review vol. 20, p. I20, 1905. 
Queen self regulating tube used for excitation was about twenty centimeters behind the screen. Roentgen rays of moderate "hardness" were used. No systematic experiments to determine the effect of varying hardness upon the form of the luminescence spectrum have yet been made, but a few preliminary tests indicate that the effect is not great. Corrections due to fluorescence excited in the glass of the spectrophotometer, which we had at first thought would be necessary, were found to be negligible.

The upper curve in Fig. 30 shows the luminescence spectrum observed during excitation, while the lower curve shows the distribution of intensity in the phosphorescence spectrum, observed $5 \mathrm{sec}$. after excitation had ceased. Owing to the weakness of the phosphorescence excited by Roentgen rays, the latter curve was determined with some difficulty, the readings at the red end of the spectrum being especially uncertain. The indications of a second maximum in the phosphorescence spectrum at $0.62 \mu$ or beyond are therefore not to be regarded as entirely trustworthy.

\section{Photo-luminescence During Excitation.}

In the experiments upon photo-luminescence during excitation the carbon arc was first used as an exciting source. The arrangement of apparatus is shown in Fig. $3 \mathrm{I}$. The zinc sulphide screen was

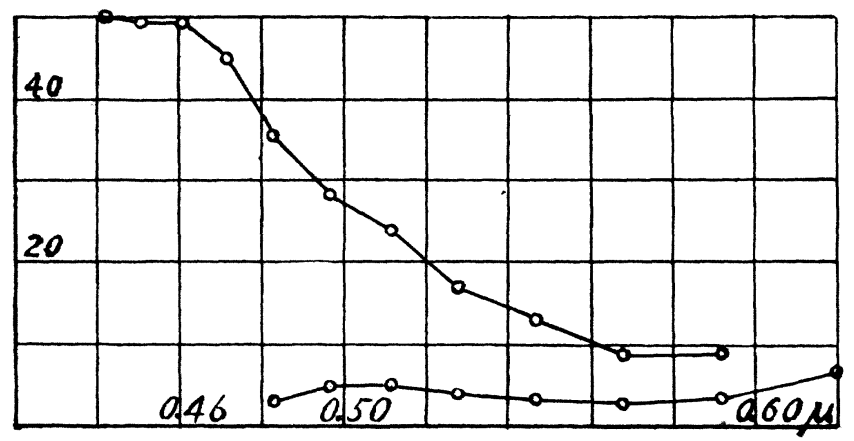

Fig. 30. The upper curve shows the luminescence spectrum of Sidot blende during excitation by Roentgen rays. The lower curve shows the phosphorescence 5 seconds after excitation.

placed in a light tight box $B$, and the exciting light from an $\operatorname{arc} A$, after dispersion by the pris $\mathrm{m} P$, entered the box through an open 
ing at the left. A second opening in $B$ allowed the fluorescence light to enter the slit of the Lummer-Brodhun spectrophotometer

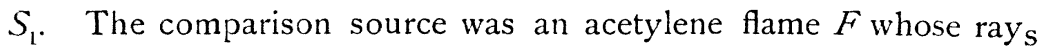
were reflected into the comparison slit by the block of magnesium carbonate, $M$. The second spectrophotometer, $S_{2}$, and the shutter $C$ were used in later experiments on phosphorescence, but not in the experiments now considered.

Luminescence $\mathrm{w}$ as excited only by the rays $a^{t}$ the violet end of the

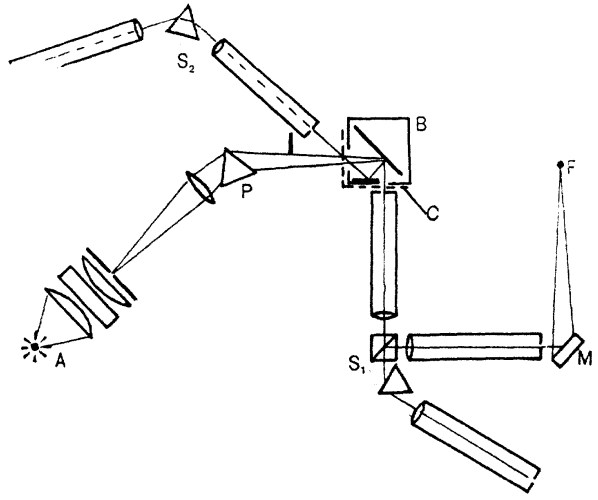

Fig. 31. arc spectrum, and especially in the region of the violet bands. $\mathrm{I} t$ is to be noted that on account of the absorption of the glass

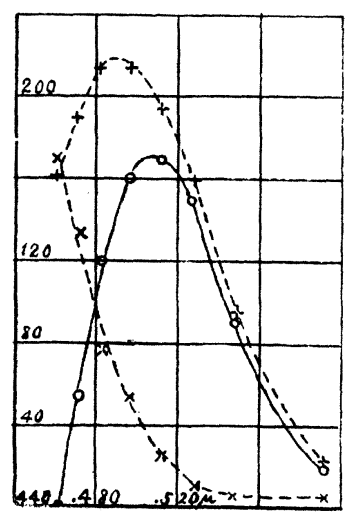

Fig. 32. Luminescence spectrum of Sidot blende during excitation by the violet bands in the arc. prism and lenses the spectrum extended only a short distance into the ultra violet.

Observations taken to determine the luminescence spectrum when the zinc sulphide screen was excited by the violet bands of the arc are plotted in Fig. 32. Owing to the impurity of the exciting spectrum a certain amount of blue and green light reached the screen even when the attempt was made to excite by violet only. Since this stray light was in part reflected into the slit of the spectrophometer with the luminescence light of the same color, it was necessary to make a correction to remove the error due to this cause. The cor-

rection was determined by replacing the zinc sulphide screen by a block of magnesium carbonate, whose surface was of approximately the same color and roughness as that of the fluorescent screen, and by measuring the light received from the magnesia 
at different points throughout the spectrum. The results of such a series of measurements is shown in the lower broken line in the figure. It will be noticed that the correction is inappreciable for the longer wave-lengths, but becomes important in the violet. The upper broken line in Fig. 32 shows the intensity of the light reaching the spectrophotometer from the zinc sulphide screen, being the combined effect of luminescence and reflected light; and the heavy curve, obtained by subtracting the ordinates of the lower curve from those of the upper, shows the corrected luminescence spectrum during excitation.

The curves in Fig. 32, like those published in our previous papers on luminescence, are expressed in terms of the acetylene flame as a standard. In other words, each ordinate represents the ratio of the intensity of the luminescence light, of the particular wave-length considered, to the intensity of the light of the same wave-length from an acetylene flame; the curves are not energy curves. Since, however, the distribution of energy in the visible spectrum of the acetylene flame is now known, ${ }^{1}$ curves showing the energy distribution in luminescence spectra may readily be computed if desired.

When the screen was excited by the arc as described above no trace of the violet luminescence, which had been so prominent with Roentgen ray excitation, could be observed. It seemed not unlikely, however, that the absence of the violet band was due to the fact that the ultraviolet rays suitable for exciting it had been removed from the light from the arc by the glass prism and lenses of the dispersing system. We therefore rearranged the apparatus so as to use a quartz prism and quartz lenses, while a spark between metal terminals was substituted for the arc. Since the excitation in the region studied was solely by ultraviolet rays that were incapable of passing through glass, it was a simple matter to test for errors due an impure spectrum, or to any other source of stray light, by inserting a piece of glass in the path of the exciting rays. This test showed that stray light was in no case present in appreciable amount.

${ }^{1}$ Nichols, Standards of Light, Proc. International Electrical Congress at St. Louis, 1904. Physical Review, vol. XXI., p. 147, 1905. 
This ultraviolet excitation developed strong fluorescence in the extreme violet, similar in color to that produced by Roentgen rays. But the green band that had been excited by the visible rays of the arc was relatively very weak. In spite of its faintness, however, the presence of the green band in the luminescence light could be readily detected, for while the violet luminescence died out almost immediately when excitation ceased, the green persisted as a slowly decaying phosphorescence observable for several minutes.

The similarity between the effects of ultraviolet light and those of Roentgen rays as exciting sources is worthy of note. In each case the chief luminescence is in the extreme violet, and is of short duration. But in each case also this is accompanied by luminescence in the green, which is relatively faint but of long duration. As is illustrated in numerous other cases, the Roentgen rays, when comparable at all with rays of light, are rather to be compared with ultraviolet light than with the rays of the visible spectrum.

The agreement between the luminescence spectrum excited by Roentgen rays and that observed in the case of ultraviolet excitation is not exact. We are of the opinion that the difference is due

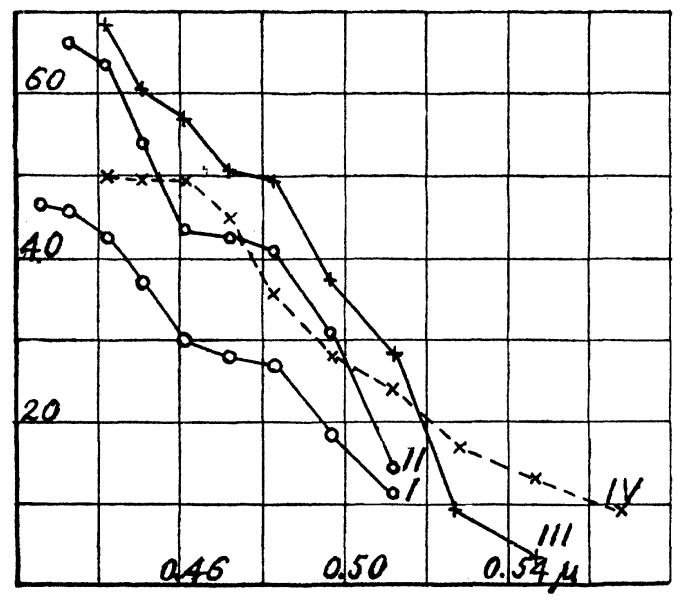

Fig. 33. Fluorescence spectrum of Sidot blende when excited by the ulıra-violet rays of the iron spark (Curves I. and II.), by the ultra-violet of the magnesium spark (Curve III.), and by the Roentgen Rays (Curve IV.).

to the fact that the violet luminescence does not consist of one band only, but of at least two, which are excited by Roentgen rays and 
ultraviolet light in different relative intensity. The matter will be made clear by reference to Fig. 33. Curve I. shows the luminescence spectrum when ultraviolet rays from the iron spark were used for excitation. The rays used were those giving most intense luminescence, and did not lie much beyond the edge of the visible spectrum. The shape of this curve suggests that it is made up of two overlapping bands, one having a maximum not far from $0.48 \mu$, while the other has its maximum near $0.42 \mu$. In Curve II. the exciting light was at the extreme ultra end of the iron spectrum, and in Curve III. the magnesium lines near $0.33 \mu$ were used in excitation. In these curves also there is every indication that the spectrum consists of at least two bands.

Unfortunately we have not been able to separate the bands more completely. It is to be remembered that the resolving power of a spectrophotometer is at the best small, and that in experiments of the kind here described the small intensity of the light studied prohibits the use of a narrow slit. Luminescence bands may sometimes be separated by a proper choice of the exciting light. In the case of Sidot blende the green band is very readily obtained alone by using the carbon bands of the arc for excitation. But the two violet bands in the luminescence of this substance are so close together that their regions of excitation appear to overlap throughout nearly their whole extent. The most that we were able to do was to show that ultraviolet rays of different wave-length produced different relative intensities in the two bands, as illustrated by Curves I. and III. of Fig. 33 .

The broken line, Curve IV. of Fig. 33 shows the luminescence spectrum excited by Roentgen rays. This is the same curve that appears in Fig. 30, and is introduced in Fig. 33 to make possible convenient comparison of the effects of ultraviolet light and Roentgen rays. It will be observed that each of the curves in Fig. 33 might well result from the superposition of three bands, whose maxima lie approximately at $0.42 \mu, 0.48 \mu$ and $0.5 \mathrm{I} \mu$. In the Roentgen ray luminescence the green band is relatively stronger than in the case of excitation by ultra-violet light, while the band at $0.48 \%$ appears to be entirely absent. In the luminescence produced by the magnesium spark (Curve III.) the green band, while still observable, is not so 
strong as in Curve IV., while the band at $048 \mu$ is readily detected. Curves I. and II. (iron spark) show scarcely any trace of the green band, but the bands at $0.48 \mu$ and at $0.42 \mu$ are well marked.

\section{Failure of Stokes' Law.}

As already stated, the green band is most brilliantly excited by the violet, although rays from all parts of the ultraviolet spectrum are also capable of producing a considerable effect. We thus see that there is the same general relation between the position of the luminescence spectrum and that of the exciting light as in the case of fluorescence. In all the cases of fluorescence thus far studied we have found that the two spectral regions overlap. It therefore seemed interesting to see whether the same is true in the present case.

To settle this point it was necessary to use in excitation a far purer spectrum than that employed in our other experiments with Sidot blende. The rather crude dispersing system shown in Fig. 3 I was therefore replaced by a large spectrometer. A spectrum of the arc was thrown on the collimator slit by the aid of a prism and single lens, and after a second dispersion in the spectrometer, light reached the phosphorescent screen as a sharply focussed band. Using the shutter described below so as to observe the phosphorescence immediately after the exciting light was cut off, and making observations with the unaided eye instead of with the spectrophotometer, it was found that phosphorescence was unquestionably present for exciting light lying between $0.470 \mu$ and $0.497 \mu$. Since the phosphorescence spectrum can readily be followed beyond $0.46 \mu$ there appears to be the same violation of Stokes' law in the phosphorescence of Sidot blende that we have previously found in the case of fluorescence.

\section{Phosphorescence Spectrum During Decadence.}

Although the phosphorescence of Sidot blende can be detected in a dark room for several hours after excitation has ceased, it remains sufficiently bright for spectrophotometric measurement only for a few seconds. In order to determine the law of decay for different 
wave-lengths and especially to determine the change, if any, in the phosphorescence spectrum during decay, the following procedure was followed:

A shutter, shown by the broken line $C$ in Fig. $3 \mathrm{I}$, and sliding upon vertical guides, was attached to the box containing the phosphorescent screen. When this shutter was raised it permitted the exciting light to enter the box, as in the experiments just described, but at the same time closed the opening in front of the collimator slit. When the shutter was dropped it first cut off the exciting light and then, a moment later, opened the window in front of the collimator. The observer was in this way protected from the brilliant luminescence produced during excitation, but was enabled to observe the phosphorescence immediately after excitation had ceased. The comparison slit being set to some suitable reading, the observer recorded upon a chronograph the instant when the two parts of the spectrophotometer field appeared equally bright. By means of a suitable electric contact on the shutter a record was also made at the time when the exciting light was cut off. In this way the time required for the phosphorescent light to fall from its initial value to any given final value could be conveniently measured.

The determination of the instant when the rapidly changing phosphorescence became equal to the constant comparison light would at first appear to be a difficult observation, and one not capable of great accuracy. As a matter of fact these observations were fully as reliable as ordinary spectrophotometric settings, and at these low intensities far less trying to the eye.

The most serious difficulty encountered in these measurements arose from the unsteadiness of the exciting light. When constancy is essential the arc, even under the best of conditions, leaves much to be desired. After numerous unsuccessful attempts to obtain steady conditions we abandoned all special efforts to keep the excitation constant, and arranged to make observations only when the exciting light, either by adjustment or by accidental fluctuation, had reached a certain definite intensity. In order to accomplish this a second spectrophotometer $\left(S_{2}\right.$ in Fig. 3I) was used. Light from the luminescent screen was thrown into the collimator by means of a mirror as shown in the figure. The comparison light came 
from the acetylene flame $F$ after reflection from a mirror and a block of magnesium carbonate not shown in the figure. One observer at the eyepiece of $S_{2}$ waited until the intensity of fluorescence reached such a value as to give equality of the two fields, and then, with suitable warning, dropped the shutter $C$. The second observer at $S_{1}$ then made a chronograph record as before described. Numerous determinations, often twenty or more, were made in this way for each point of the curves described below.

A preliminary set of measurements in which the duration of excitation was varied from $3 \mathrm{sec}$. to $30 \mathrm{sec}$. showed no variation in the time of decay. We conclude that the full effect of the exciting light is produced in less than $3 \mathrm{sec}$. In the subsequent experiment the duration of excitation was never less than $5 \mathrm{sec}$.

TABLE I.

Giving the observed time, in seconds, during which the phosphorescence fell from its initial intensity to the intensity given at the top of the column.

\begin{tabular}{c|l|l|l|l|l}
\hline Wave-length. & $I=$ 100. & $I=60$. & $I=0$. & $I=$ 10. & $I=5$. \\
\cline { 1 - 2 } $0.461 \mu$ & & & & 0.458 & \\
0.472 & & 0.00 & & 0.99 & \\
0.483 & 0.00 & 0.456 & 0.745 & 2.07 & 7.1 \\
0.497 & 0.491 & 0.95 & 1.63 & 3.13 & \\
0.512 & 0.502 & 1.00 & 1.80 & 3.41 & 10.4 \\
0.528 & 0.290 & 0.682 & 1.49 & 2.91 & \\
0.535 & 0.00 & & & & \\
0.547 & & 0.223 & 0.838 & 2.55 & 7.5 \\
0.553 & & 0.00 & & & \\
0.568 & & & 0.302 & 1.30 & \\
0.572 & & & 0.00 & & \\
0.592 & & & & 0.713 & 4.1 \\
\hline
\end{tabular}

The results of one set of determinations made in this way are given in Table I. and are plotted in Fig. 34. In Curve I. the width of the comparison slit was kept at IoO divisions of its micrometer screw, and the time required for the phosphorescence of any given wave-length to fall from its initial value to this intensity was determined as described above. The curve is obtained by plotting wave-lengths as abscissas and times as ordinates. In Curve II. the width of the comparison slit was 60 divisions; in Curve III., 30 divisions; and in Curve IV., Io divisions. Obser- 
vations made at 5 divisions are not included on the plot. Points lying on the horizontal axis in Fig. 34 were located by determining

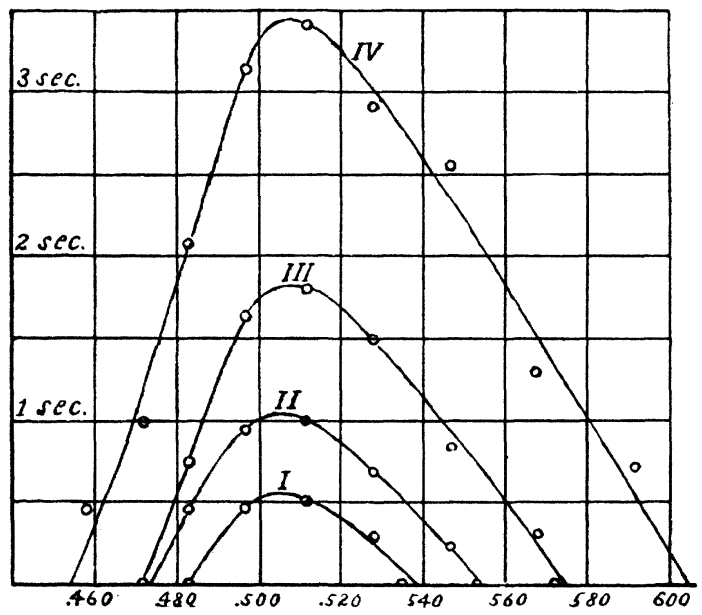

Fig. 34. Curves showing the time required for the phosphorescence to fall from its initial intensity to a given final intensity. The final intensity is kept constant throughout each curve.

by trial the wave-length for which there was a balance in the spec-

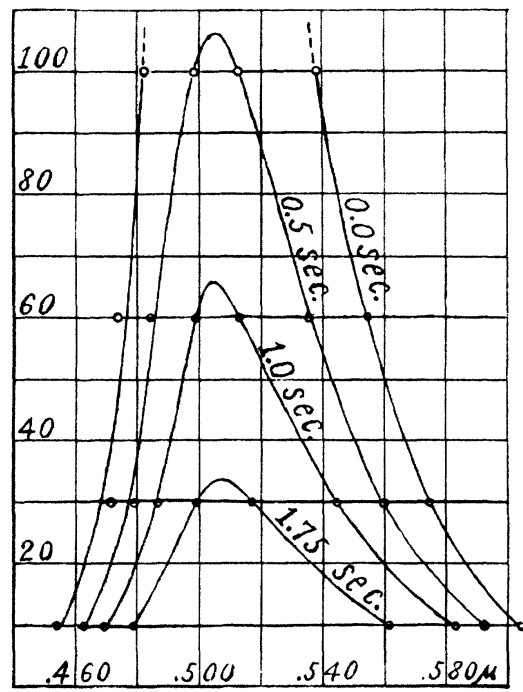

Fig. 35. The phosphorescence spectrum of Sidot blende at different times after the removal of the exciting light. trophotometer the instant after dropping the shutter. These points are probably somewhat less reliable than the others.

The significance of these results is better shown by plotting them in a different way. In Fig. 35 wave-lengths are plotted as abscissas as before, but intensities, instead of times, are plotted as ordinates. Fach curve in this figure shows the distribution of intensity in the phosphorescence spectrum at some definite time after the removal of the exciting light.

No curves have been plotted for intervals of more than 
I.75 sec. after excitation had ceased, since our data furnished so few points for the later curves that their form would be largely a matter of conjecture. ${ }^{1}$ As already stated, however, a curve of the type shown in Fig. 34 was determined for an intensity of 5 divisions. The maximum ordinate of this curve was IO.I sec. and occurred at $0.506 \mu-i . e$. , at the same wave-length as in the case of the curves shown in 34. Comparison with Fig. 32 shows that the maximum of the luminescence spectrum during excitation also occurs at this wave-length. In the case of the green band of Sidot blende we conclude therefore that the maximum of the fluorescence spectrum occurs at the same wave-length as that of the phosphorescence spectrum; and that no change that could be detected by our apparatus occurs in the position of this maximum for ten seconds after excitation has ceased.

Fig. 35 also shows that if any change occurs in the form of the phosphorescence spectrum during decadence, this change is extremely small. In other words the different wave-lengths of the phosphorescent spectrum decay at the same rate. Table II. will show to what extent this conclusion is justified by the data. Each column of this table refers to the wave-length stated at the top, and in it are tabulated the intensities for this wave-length at different intervals after the end of excitation, these intensities being expressed in terms of the intensity at the end of $1.75 \mathrm{sec}$. as unity. If the phosphorescence spectrum remains absolutely unaltered during decay, all the numbers in this table that lie in a given horizontal line should be equal.

TABLE II.

Intensity of Phosphorescence at Different Intervals After Excitation Had Ceased, Expressed in Terms of the Intensity at the End of $1.75 \mathrm{Sec}$.

\begin{tabular}{|c|c|c|c|c|c|}
\hline & $0.4^{8 \mu} \mu$ & $0.50 \mu$ & $0.52 \mu$ & $0.54 \mu$ & $0.5^{6} \mu$ \\
\hline 0.0 sec. & $7.8 ?$ & & & 5.2 & 4.8 \\
\hline 0.5 “ & 3.5 & 3.3 & 3.1 & 3.0 & 2.8 \\
\hline $1.0 "$ & 1.9 & 2.0 & 1.8 & 1.8 & 1.9 \\
\hline 1.75 ، & 1.0 & 1.0 & 1.0 & 1.0 & 1.0 \\
\hline
\end{tabular}

${ }^{1}$ In a series of observations of the kind recorded in Fig. 34 the number of points that could be located was limited both by the difficulty of maintaining constant conditions during the three or four hours of observation, and by the endurance of the observer's eye. 
The numbers in Table II. corresponding to o.o sec. are uncertain ; first, because the observations by which the initial phosphorescence was determined were themselves especially uncertain; and second, because the steepness of the curve (Fig. 35) in the neighborhood of $0.48 \mu$ and $0.54 \mu$ would cause a slight change in the manner of plotting to produce a large change in the ordinates at these wave-lengths. If the ratios tabulated for $0.0 \mathrm{sec}$. are left out of consideration, the agreement between the remaining ratios, at 0.5 sec. and I.O sec. is as close as could be expected. We conclude that, although there is some indication of more rapid decadence at the ultra edge of the phosphorescence spectrum, the probability is that all parts of the spectrum decay at the same rate, and that the form of the phosphorescence spectrum remained unchanged.

While the result obtained in this one case is not sufficient to establish a general law, we are nevertheless of the opinion that the behavior of Sidot blende is typical, and that in no case of phosphorescence is there any change in the form of the spectrum during decadence. In complex cases of phosphorescence we do not imply by this that the phosphorescence spectrum as a whole remains unchanged in form, but rather that the distribution of intensity in each band is unaltered. If the phosphorescence consists of several bands, it is to be expected that the different bands will decay at different rates. In fact Sidot blende itself furnishes an extreme illustration of this, for the violet bands die out in one or two tenths of a second, while the green band persists for hours.

Numerous cases in which the color of a phosphorescent substance seems to change as the phosphorescence dies out at first appear to contradict this view absolutely. We think, however, that all cases of this kind may be shown to belong to one of the two following classes:

I. Cases of real color change. For example, anisic acid at low temperatures, where the phosphorescence changes from blue to greenish yellow. ${ }^{1}$ Such cases are probably due to the presence in the luminescence spectrum of several phosphorescence bands, which decay at different rates. In the case of anisic acid the results would be explained by the presence of a brilliant but rapidly decaying 
band in the blue, and a persistent band of smaller initial intensity in the yellow.

2. Cases where the apparent change in color is due to the fact that the color sense in the eye is either weak or entirely absent at low intensities. At very low intensities all colors appear to the eye as gray. However brilliant may be the color of the phosphorescence light initially, it loses this color and changes to a gray or faint white as it becomes fainter. But such a change as this is in the retina, and does not indicate any change in the phosphorescence spectrum. We are convinced that the use of the unaided eye in the study of luminescence may lead to serious errors.

It is interesting to note that attention was called to both of these causes of change of color during decay by the elder Becquerel. ${ }^{1}$

The data of Table I. might also be used to study the law of decay of phosphorescence, $i$. $e$, the relation between intensity and time. More reliable results are obtained, however, by studying one wave-length at a time, since the data necessary for plotting a decadence curve may in this case be obtained more quickly and are, therefore, less liable to error due to fluctuating conditions. Experiments dealing with the law of decadence and with the effect of infra-red radiation upon phosphorescence will be described later.

${ }^{1}$ Ed. Becquerel, C. R., vol. 49, p. 27, 1859; Ann. de Chemie et de Physique. Series 4, vol. 62, p. 20, 1861 . 